\title{
MULTI-PERIOD MEAN-VARIANCE PORTFOLIO OPTIMIZATION WITH MARKOV SWITCHING PARAMETERS
}

\author{
Oswaldo L. V. Costa* \\ oswaldoelac.usp.br
}

\author{
Michael V. Araujo* \\ michael.araujo@poli.usp.br
}

* Departamento de Engenharia de Telecomunicações e Controle

Escola Politécnica da Universidade de São Paulo

CEP: 05508-900, São Paulo, SP, Brasil

\begin{abstract}
In this paper we deal with a multi-period mean-variance portfolio selection problem with the market parameters subject to Markov random regime switching. We analytically derive an optimal control policy for this mean-variance formulation in a closed form. Such a policy is obtained from a set of interconnected Riccati difference equations. Additionally, an explicit expression for the efficient frontier corresponding to this control law is identified and numerical examples are presented.
\end{abstract}

KEYWORDS: optimal control, Markov chain, stochastic systems, portfolio optimization, multi-period mean-variance.

\section{RESUMO}

Investiga-se um modelo multi-dimensional de seleção de carteiras em média-variância, no qual os parâmetros de mercado estão sujeitos a saltos Markovianos. Deriva-se analiticamente uma estratégia de controle ótima em forma fechada para esta formulação de média-variância. Esta estratégia é obtida através de um conjunto de equações a diferenças de Riccati. Adicionalmente, uma expressão explícita para a fronteira eficiente correspondente a este controle ótimo é identificada e exemplos numéricos são apresentados.

\footnotetext{
Artigo submetido em 24/05/2007

1a. Revisão em 04/06/2007

2a. Revisão em 12/03/2008

Aceito sob recomendação do Editor Associado

Prof. José Roberto Castilho Piqueira
}

PALAVRAS-CHAVE: controle ótimo, cadeia de Markov, sistemas estocásticos, otimização de portfólio, média-variância em multi-período.

\section{INTRODUCTION}

Since the seminal work of (Markowitz, 1952), the research on the mean-variance approach to portfolio selection has increased in order to provide financial models with more realistic assumptions. One of the main advantages of the meanvariance criterion is that it has a simple and clear interpretation in terms of individual portfolio choice and utility optimization, although some of its drawbacks are nowadays well known. The original Markowitz's formulation aims at selecting a single portfolio which yields the greatest expected final wealth subject to a maximal final risk level (terminal wealth variance), or to find the single portfolio which produces the smallest terminal wealth variance subject to minimal final expected wealth. Recently (see ( $\mathrm{Li}$ and $\mathrm{Ng}, 2000)$ ) these two formulations were extended into a multi-period framework, in which the objective is to select a set of intermediate portfolios instead of just one as originally proposed (see the mathematical formulation of these problems in (4) and (5)). When applying dynamic programming to analytically solve these multi-period mean-variance problems a technical difficulty arises due to the existence of a nonlinear term of the form $U(E(\cdot))$ (or specifically $E(V(T))^{2}$ ), where $U$ is nonlinear utility function, in the objective function. This difficulty was overcome in ( $\mathrm{Li}$ and $\mathrm{Ng}, 2000)$ by the introduction of an auxiliary problem. 
In ( $\mathrm{Li}$ and $\mathrm{Ng}, 2000)$ the market uncertainties are reproduced by stochastic models in which the key parameters, expected return and volatility, are deterministic. As stated by (Zhang, 2000), such models are good only for a short period since they would not respond appropriately to random changes in these parameters due to some sudden market discontinuities (for example, the one caused by a terrorist strike). As a result, there has been an increasing interest in the study of financial models in which the key parameters are modulated by a Markov chain, see for instance (Bauerle and Rieder, 2004), (Yin and Zhou, 2004), (Zhang, 2000), (Zhou and Yin, 2003) and (Çakmak and Özekici, 2006). Indeed, such models can better reflect the market environment as the overall assets usually move according to a major trend given by the state of the underlying economy or by the general mood of the investors.

In (Çakmak and Özekici, 2006) and in (Yin and Zhou, 2004), discrete-time models for the mean-variance portfolio selection problem with Markov switching were considered. It is important to stress the main differences between our work and these works. The basic idea in (Yin and Zhou, 2004) is to use the optimal strategy of the limit continuous time problem obtained in (Zhou and Yin, 2003) to derive a nearly optimal portfolio for the discrete time model presented in equation (6) of (Yin and Zhou, 2004). In (Çakmak and Özekici, 2006), the authors adopt a more direct approach to tackle the problem, avoiding any kind of approximating assumption as required in (Yin and Zhou, 2004), and obtaining optimal results, instead of nearly optimal as in (Yin and Zhou, 2004). However, all assets in the financial market considered in (Çakmak and Özekici, 2006), including the risk free one, depend on a Markov chain. In our paper we follow a direct approach as in (Çakmak and Özekici, 2006), extending their work in two other directions. First we consider a financial model more general than that in (Çakmak and Özekici, 2006), in which all assets are risky and dependent of a Markov chain. After that we consider a financial model in which there is a riskless asset independent of any source of uncertainty, even the Markov chain, and the risky ones, which depend on a Markov chain. In this case more specific and interesting results can be analytically derived for the mean-variance portfolio selection problem with regime switching.

This paper is organized as follows. In Section 2 we formulate the model and the problems to be investigated. In Section 3, an optimal control policy for an auxiliary problem as well as the expected value and variance of the terminal wealth are analytically derived. Such a policy can be obtained by the solution of a set of interconnected Riccati difference equations. The solution of the mean-variance problems and an explicit expression for the efficient frontier are derived in Section 4. The case in which there is a riskless asset is considered in
Section 5. Numerical examples are presented in Section 6. The paper is concluded in Section 7 with some final remarks.

\section{PROBLEM FORMULATION}

Throughout the paper we shall denote by $\mathbb{R}^{n}$ the $n$ dimensional Euclidean real space and by $\mathbb{R}^{n \times m}$ the Euclidean space of all $n \times m$ real matrices. For a sequence of numbers $a_{1}, \ldots, a_{m}$, we shall denote by $\operatorname{diag}\left(a_{i}\right)$ the diagonal matrix in $\mathbb{R}^{m \times m}$ formed by the element $a_{i}$ in the $i^{t h}$ diagonal, $i=1, \ldots, m$. The superscript ' will denote the transpose of a vector or matrix. We will consider a financial market with $n+1$ risky securities on a complete filtered probability space $\left(\Omega, \mathcal{F},\left\{\mathcal{F}_{t}\right\}, \mathcal{P}\right)$. The assets' price will be described by the random vector $\overline{\mathcal{S}}(t)=\left(\mathcal{S}_{0}(t), \ldots, \mathcal{S}_{n}(t)\right)^{\prime}$ taking values in $\mathbb{R}^{n+1}$ with $t=0, \ldots, T$. Set $\overline{\mathcal{R}}(t)=$ $\left(\mathcal{R}_{0}(t), \ldots, \mathcal{R}_{n}(t)\right)^{\prime}$, with $\mathcal{R}_{i}(t)=\frac{S_{i}(t+1)}{S_{i}(t)}$. We assume that the random vector $\overline{\mathcal{R}}(t)$ satisfies the following equation:

$$
\overline{\mathcal{R}}(t)=[\bar{e}+\bar{\mu}(t, \theta(t))]+\bar{\sigma}(t, \theta(t)) W(t),
$$

where $\bar{e}=(1, e)^{\prime}$, with $e \in \mathbb{R}^{n}$ a vector with $1^{\prime} s$ in all its components. Here $\{\theta(t) ; t=0, \ldots, T\}$ is a finite-state discrete-time Markov chain with state space $\mathcal{M}=\{1, \ldots, m\}$, and $\{W(t) ; t=0, \ldots, T\}$ is a sequence of $(n+1)$-dimensional independent random vectors with zero mean and covariance $I$ (identity matrix). We assume that $\{W(t), \theta(t)\}$ are mutually independent. The set $\mathcal{M}$ represents the possible operations mode of the market. $\mathcal{P}$ is a probability measure such that $\mathcal{P}(\theta(t+1)=j \mid \theta(0), \ldots, \theta(t)=i)=$ $\mathcal{P}(\theta(t+1)=j \mid \theta(t)=i)=p_{i j}(t), p_{i j}(t) \geq 0$ and $\sum_{j \in \mathcal{M}} p_{i j}(t)=1$, for $t=0, \ldots, T-1$ and $i, j \in \mathcal{M}$. We set for $t=0, \ldots, T, P(t)=\left[p_{i j}(t)\right]_{m \times m}, \pi_{i}(t)=$ $\mathcal{P}(\theta(t)=i), \pi(t)=\left(\pi_{1}(t), \ldots, \pi_{m}(t)\right)^{\prime}$. As in (Costa et al., 2005), for $z=\left(z_{1}, \ldots, z_{m}\right)^{\prime} \in \mathbb{R}^{m}$, we define the operator $\mathcal{E}(z, t)=\left(\mathcal{E}_{1}(z, t), \ldots, \mathcal{E}_{m}(z, t)\right)$ as $\mathcal{E}_{i}(z, t)=$ $\sum_{j=1}^{m} p_{i j}(t) z_{j}$, for $i \in \mathcal{M}$. For notational simplicity, we shall omit from now on the variable $t$ in $p_{i j}(t)$ and $\mathcal{E}_{i}(z, t)$. The filtration $\mathcal{F}_{t}$ is such that the random vectors $\{\overline{\mathcal{S}}(k) ; k=$ $0, \ldots, t\}$ and Markov chain $\{\theta(k) ; k=0, \ldots, t\}$ are $\mathcal{F}_{t^{-}}$ measurable.

When the market operation mode is $\theta(t)=i \in \mathcal{M}$, $\bar{\mu}(t, i) \in \mathbb{R}^{n+1}$ represents the vector with the expected returns of the assets, while $\bar{\sigma}(t, i) \bar{\sigma}(t, i)^{\prime} \in$ $\mathbb{R}^{(n+1) \times(n+1)}$ is the covariance matrix of the returns. It will be convenient to decompose $\bar{\mu}(t, i)$ and $\bar{\sigma}(t, i)$ as $\bar{\mu}(t, i)=\left[\begin{array}{c}\mu_{0}(t, i) \\ \mu(t, i)\end{array}\right]$ and $\bar{\sigma}(t, i)=\left[\begin{array}{c}\sigma_{0}(t, i) \\ \sigma(t, i)\end{array}\right]$, with $\mu(t, i)=\left(\mu_{1}(t, i), \ldots, \mu_{n}(t, i)\right)^{\prime} \in \mathbb{R}^{n}, \sigma_{0}(t, i)=$ $\left(\sigma_{00}(t, i), \ldots, \sigma_{0 n}(t, i)\right) \in \mathbb{R}^{1 \times n+1}$, and $\sigma(t, i)=$ $\left[\sigma_{\ell, j}(t, i)\right] \in \mathbb{R}^{n \times n+1}$. We shall assume in this paper that 
$E\left(\overline{\mathcal{R}}(t) \overline{\mathcal{R}}(t)^{\prime} \mid \theta(t)=i\right)>0$, for each $t=0, \ldots, T-1$ and $i \in \mathcal{M}$.

The set of admissible investment strategies $\mathcal{U}=\{u=$ $(u(0), \ldots, u(T-1))\}$ is such that for each $i=0, \ldots, n$ and $t=0, \ldots, T-1, u(t)=\left(u_{1}(t), \ldots, u_{n}(t)\right)^{\prime}$, is a $\mathcal{F}_{t}$-measurable random vector taking values in $\mathbb{R}^{n}$. We have that $u(t)$ represents the amount of the wealth allocated among the $n$ securities. Associated to each admissible investment strategy $u$ we have the portfolio's value process $\left\{V^{u}(t) ; t=0, \ldots, T-1\right\}$, which represents the investor's wealth at the end of time $t$. For notational simplicity, we shall suppress the superscript ${ }^{u}$ whenever no confusion may arise. Assuming that the initial wealth $V(0)=V_{0}>0$ and that the portfolio is self-financed, the wealth process is represented by (see, for instance, $(\mathrm{Li}$ and $\mathrm{Ng}, 2000)$ ):

$$
\begin{aligned}
V(t+1) & =V(t)\left[1+\mu_{0}(t, \theta(t))+\sigma_{0}(t, \theta(t)) W(t)\right] \\
& +u(t)^{\prime}\left[\mu(t, \theta(t))-e \mu_{0}(t, \theta(t))\right. \\
& \left.+\left(\sigma(t, \theta(t))-e \sigma_{0}(t, \theta(t))\right) W(t)\right]
\end{aligned}
$$

Note that the amount of wealth allocated to the asset $i=0$ is determined by $V(t)-e^{\prime} u(t)$. Defining $\bar{A}_{\theta(t)}(t)=1+$ $\mu_{0}(t, \theta(t)), \widetilde{A}_{\theta(t)}(t)=\sigma_{0}(t, \theta(t)), \bar{B}_{\theta(t)}(t)=\mu(t, \theta(t))-$ $e \mu_{0}(t, \theta(t))$, and $\widetilde{B}_{\theta(t)}(t)=\sigma(t, \theta(t))-e \sigma_{0}(t, \theta(t))$, we can rewrite (2) as:

$$
V(t+1)=A_{\theta(t)}(t) V(t)+B_{\theta(t)}(t)^{\prime} u(t),
$$

where

$$
A_{\theta(t)}(t)=\bar{A}_{\theta(t)}(t)+\widetilde{A}_{\theta(t)}(t) W(t)
$$

and

$$
B_{\theta(t)}(t)=\bar{B}_{\theta(t)}(t)+\widetilde{B}_{\theta(t)}(t) W(t) .
$$

The multi-period mean-variance problem aims at selecting $u \in \mathcal{U}$ which has the greatest expected terminal wealth given an affordable terminal wealth variance, or which produces the lesser variance of the final wealth given a desirable expected terminal wealth. Formally these problems, named respectively $P 1\left(\sigma^{2}\right)$ and $P 2(\epsilon)$, can be posed as:

$$
\begin{gathered}
P 1\left(\sigma^{2}\right): \min _{u \in \mathcal{U}}-E(V(T)) \\
\text { subject to }: \operatorname{Var}(V(T)) \leq \sigma^{2} \\
P 2(\epsilon): \min _{u \in \mathcal{U}} \operatorname{Var}(V(T)) \\
\text { subject to }: E(V(T)) \geq \epsilon
\end{gathered}
$$

Alternatively, an unconstrained form would be:

$$
P 3(\nu): \min _{u \in \mathcal{U}} \nu \operatorname{Var}(V(T))-E(V(T)),
$$

where $\nu \in[0, \infty)$ represents the investor's risk aversion coefficient, giving his trade-off preference between the expected terminal wealth and the associated risk level. Due to the difficulty in solving directly the three problems above we shall consider, as in ( $\mathrm{Li}$ and $\mathrm{Ng}, 2000$ ), an auxiliary formulation. Using the fact that $\nu \operatorname{Var}(V(T))-E(V(T))=$ $\nu E\left(V(T)^{2}\right)-\left[\nu E^{2}(V(T))+E(V(T))\right]$, we can associate to $P 3(\nu)$ the following auxiliary problem:

$$
A(\lambda, \nu): \min _{u \in \mathcal{U}} E\left\{\nu V(T)^{2}-\lambda V(T)\right\} .
$$

\section{OPTIMAL CONTROL POLICY FOR THE AUXILIARY PROBLEM}

In this section we obtain an explicit expression for the value function and optimal control policy for the auxiliary problem $A(\lambda, \nu)$ by applying dynamic programming. We also obtain closed expressions for the expected value and variance of the terminal wealth. As in the classical stochastic linear quadratic problem, this optimal control law depends on the solution of a set of recursive coupled Riccati difference equations (see (8) below). Before going to the main result, let us define some intermediate problems. The value function for the auxiliary problem at time $k \in\{0, \ldots, T-1\}$ is defined by:

$$
J(V(k), \theta(k), k)=\min _{u_{k} \in \mathcal{U}_{k}} E\left\{\nu V(T)^{2}-\lambda V(T) \mid \mathcal{F}_{k}\right\},
$$

where $\mathcal{U}_{k}=\left\{u_{k}=(u(k), \ldots, u(T-1))^{\prime} ; u(t)\right.$ is $\mathcal{F}_{t}$ measurable for each $t=k, \ldots, T-1\}$. We shall need the following definitions. For each $i \in \mathcal{M}$ and $t=0, \ldots, T$, set:

$$
\begin{aligned}
\chi_{i}(t) & =E\left(B_{i}(t)\right)=\bar{B}_{i}(t), \\
\phi_{i}(t) & =E\left(B_{i}(t) B_{i}(t)^{\prime}\right)=\bar{B}_{i}(t) \bar{B}_{i}(t)^{\prime}+\widetilde{B}_{i}(t) \widetilde{B}_{i}(t)^{\prime}, \\
\delta_{i}(t) & =E\left(A_{i}(t)^{2}\right)=\bar{A}_{i}(t)^{2}+\left\|\widetilde{A}_{i}(t)\right\|^{2}, \\
\varphi_{i}(t)^{\prime} & =E\left(A_{i}(t) B_{i}(t)^{\prime}\right)=\bar{A}_{i}(t) \bar{B}_{i}(t)^{\prime}+\widetilde{A}_{i}(t) \widetilde{B}_{i}(t)^{\prime}, \\
\beta_{i}(t) & =\chi_{i}(t)^{\prime} \phi_{i}(t)^{-1} \chi_{i}(t), \\
Q_{i}(t) & =\delta_{i}(t)-\varphi_{i}(t)^{\prime} \phi_{i}(t)^{-1} \varphi_{i}(t), \\
Q(t) & =\operatorname{diag}\left(Q_{i}(t)\right), \\
R_{i}(t) & =\bar{A}_{i}(t)-\chi_{i}(t)^{\prime} \phi_{i}(t)^{-1} \varphi_{i}(t), \\
R(t) & =\operatorname{diag}\left(R_{i}(t)\right) .
\end{aligned}
$$

Notice that from the hypothesis that $E\left(\overline{\mathcal{R}}(t) \overline{\mathcal{R}}(t)^{\prime} \mid \theta(t)=i\right)>0$, the inverse of $\phi_{i}(t)$ is well defined and $Q_{i}(t)>0$. We compute backwards the $m$ dimensional vectors $K(t)=\left(K_{1}(t), \ldots\right.$, $\left.K_{m}(t)\right)^{\prime}, \quad Z(t)=\left(Z_{1}(t), \ldots, Z_{m}(t)\right)^{\prime} \quad$ and $D(t)=\left(D_{1}(t), \ldots, D_{m}(t)\right)^{\prime}$, with $K_{i}(t), Z_{i}(t)$ and $D_{i}(t)$ as follows: For $t=T-1, \ldots, 0$ and $i \in M$ :

$$
\left\{\begin{array}{l}
K_{i}(t)=Q_{i}(t) \mathcal{E}_{i}[K(t+1)], \quad K_{i}(T)=\nu, \\
Z_{i}(t)=R_{i}(t) \mathcal{E}_{i}[Z(t+1)], \quad Z_{i}(T)=-\lambda, \\
D_{i}(t)=\frac{\mathcal{E}_{i}[Z(t+1)]^{2}}{4 \mathcal{E}_{i}[K(t+1)]} \beta_{i}(t)+\mathcal{E}_{i}[D(t+1)], \quad D_{i}(T)=0 .
\end{array}\right.
$$


From (8) we have by backward iteration that

$$
\begin{aligned}
K(t) & =(Q(t) P(t) \cdot \ldots \cdot Q(T-1) P(T-1)) e \nu \\
& =\nu \mathcal{K}(t),
\end{aligned}
$$

and

$$
\begin{aligned}
Z(t) & =-(R(t) P(t) \cdot \ldots \cdot R(T-1) P(T-1)) e \lambda \\
& =-\lambda \mathcal{Z}(t)
\end{aligned}
$$

where $K(T)=\nu e$, and $Z(T)=-\lambda e$, and for $t=0, \ldots, T$, $\mathcal{K}(t) \in \mathbb{R}^{m}$ and $\mathcal{Z}(t) \in \mathbb{R}^{m}$ are defined as follows:

$\mathcal{K}(t)=\left(\prod_{k=t}^{T-1} Q(t) P(t)\right) e, \quad \mathcal{Z}(t)=\left(\prod_{k=t}^{T-1} R(t) P(t)\right) e$.

Equations (8) and (9) are related to the solution of problem (7) as stated in the next theorem.

We have the following theorem.

Theorem 1 The optimal control law for problem (7) is given by

$$
\begin{aligned}
u(t)= & -\phi_{\theta(t)}(t)^{-1} \varphi_{\theta(t)}(t) V(t) \\
& -\frac{\mathcal{E}_{\theta(t)}[Z(t+1)]}{2 \mathcal{E}_{\theta(t)}[K(t+1)]} \phi_{\theta(t)}(t)^{-1} \chi_{\theta(t)}(t)
\end{aligned}
$$

Furthermore, the value function for the intermediate problem is

$$
\begin{aligned}
J(V(t), \theta(t), t)= & K_{\theta(t)}(t) V(t)^{2}+Z_{\theta(t)}(t) V(t) \\
& +D_{\theta(t)}(t) .
\end{aligned}
$$

Proof: Let us apply induction on $t$. For $t=T$ we have that

$$
\begin{aligned}
& J(V(T), \theta(T), T)=\nu V(T)^{2}-\lambda V(T) \\
& =K_{\theta(T)}(T) V(T)^{2}+Z_{\theta(T)}(T) V(T)+D_{\theta(T)}(T),
\end{aligned}
$$

in agreement with Theorem 1. Suppose the result holds for $t=k+1$. We show next that the solution also holds for $t=k$. For $\theta(k)=i \in \mathcal{M}$ and $V(k)=v$ we have from the Bellman's principle of optimality that

$$
\begin{aligned}
J(v, i, k) & =\min _{u(k)} E\left\{J(V(k+1), \theta(k+1), k+1) \mid \mathcal{F}_{k}\right\} \\
& =\min _{u(k)} E\left\{K_{\theta(k+1)}(k+1) V(k+1)^{2}\right. \\
& +Z_{\theta(k+1)}(k+1) V(k+1) \\
& \left.+D_{\theta(k+1)}(k+1) \mid \mathcal{F}_{k}\right\} \\
& =\min _{u(k)}\left\{\mathcal { E } _ { i } [ K ( k + 1 ) ] \left[\delta(k, i) v^{2}\right.\right. \\
& \left.+2 \varphi(k, i)^{\prime} u(k) v+u(k)^{\prime} \phi(k, i) u(k)\right] \\
& +\mathcal{E}_{i}[Z(k+1)]\left[\bar{A}(k, i) v+\chi(k, i)^{\prime} u(k)\right] \\
& \left.+\mathcal{E}_{i}[D(k+1)]\right\} .
\end{aligned}
$$

Taking the derivative of (12) over $u(k)$ and making the result equal to zero yields

$$
\begin{array}{r}
2 \mathcal{E}_{i}[K(k+1)][\varphi(k, i) v+\phi(k, i) u(k)] \\
+\mathcal{E}_{i}[Z(k+1)] \chi(k, i)=0
\end{array}
$$

and from (13) we get (10). Substituting (10) into (12) yields the value function expressed in (11), providing the desired result.

Next we analytically derive expressions for the expected value and variance of the terminal wealth under the optimal control law (10). These expressions will be written in terms of some key parameters $a, b, c$ and $d$. First we make the following definitions, related to the calculation of the expected value and variance of the portfolio, and used in the proof of Theorem 2. For each $j \in \mathcal{M}$ and $t=0, \ldots, T$, define

$$
\begin{aligned}
h_{j}(t) & =\frac{1}{2} \sum_{i=1}^{m} p_{i j} \pi_{i}(t) \frac{\mathcal{E}_{i}[\mathcal{Z}(t+1)]}{\mathcal{E}_{i}[\mathcal{K}(t+1)]} \beta_{i}(t), \\
\widetilde{h}(t) & =\left(h_{1}(t), \ldots, h_{m}(t)\right)^{\prime}, \\
r_{j}(t) & =\frac{1}{4} \sum_{i=1}^{m} p_{i j} \pi_{i}(t)\left(\frac{\mathcal{E}_{i}[\mathcal{Z}(t+1)]}{\mathcal{E}_{i}[\mathcal{K}(t+1)]}\right)^{2} \beta_{i}(t), \\
\widetilde{r}(t) & =\left(r_{1}(t), \ldots, r_{m}(t)\right)^{\prime}, \\
a & =V(0) \pi(0)^{\prime} \mathcal{Z}(0), b=\sum_{k=0}^{T-1} \widetilde{h}(k)^{\prime} \mathcal{Z}(k+1) \\
c & =V(0)^{2} \pi(0)^{\prime} \mathcal{K}(0), d=\sum_{k=0}^{T-1} \widetilde{r}(k)^{\prime} \mathcal{K}(k+1) .
\end{aligned}
$$

Notice that from (14) and (15), $2 \widetilde{r}(k)^{\prime} \mathcal{K}(k+1)=$ $\widetilde{h}(k)^{\prime} \mathcal{Z}(k+1)$ and thus from (16) and (17), $2 d=b$. The constant $a$ represents the expected amount the investor obtains by investing in the reference asset $j=0$ and similarly $b$ multiplied by the ratio $\frac{\lambda}{\nu}$ yields the expected amount the investor gets by investing in the assets $j \neq 0$. The case in which $b=0$ represents the situation in which it is not worth investing in these assets, as seen in the next theorem.

Theorem 2 Under the optimal control law (10), the expected value and variance of the terminal wealth are:

$$
\begin{aligned}
E(V(T)) & =a+\frac{\lambda}{\nu} b \\
\operatorname{Var}(V(T)) & =c-a^{2}-\left(\frac{\lambda}{\nu}\right) \cdot \frac{b}{2}\left(4 a-\left(\frac{\lambda}{\nu}\right)(1-2 b)\right) .
\end{aligned}
$$

with $a, b$ and $c$ as in (16) and (17), respectively. 
Proof: First we proceed to find the expression for the expected value of the final wealth. Using the control law (10) into (3), we get

$$
\begin{aligned}
& V(t+1)=\left(A_{\theta(t)}(t)-B_{\theta(t)}(t)^{\prime} \phi_{\theta(t)}(t)^{-1} \varphi_{\theta(t)}(t)\right) \\
& \cdot V(t)-\frac{\mathcal{E}_{\theta(t)}[Z(t+1)]}{2 \mathcal{E}_{\theta(t)}[K(t+1)]} B_{\theta(t)}(t)^{\prime} \phi_{\theta(t)}(t)^{-1} \chi_{\theta(t)}(t) .
\end{aligned}
$$

Let $q_{j}(t)=E\left(V(t) 1_{\{\theta(t)=j\}}\right)$. From (Costa et al., 2005) and (20) it follows that

$$
\begin{aligned}
q_{j}(t+1) & =\sum_{i=1}^{m} p_{i j} R_{i}(t) q_{i}(t) \\
& -\frac{1}{2} \sum_{i=1}^{m} p_{i j} \pi_{i}(t) \frac{\mathcal{E}_{i}[Z(t+1)]}{\mathcal{E}_{i}[K(t+1)]} \beta_{i}(t) .
\end{aligned}
$$

Defining $\widetilde{q}(t)=\left(q_{1}(t), \ldots, q_{m}(t)\right)^{\prime}$ and replacing (14) into (21) leads to

$$
\widetilde{q}(t+1)^{\prime}=\widetilde{q}(t)^{\prime} R(t) P(t)+\frac{\lambda}{\nu} \widetilde{h}(t)^{\prime},
$$

and thus

$$
\begin{aligned}
\widetilde{q}(T)^{\prime} & =\widetilde{q}(0)^{\prime} \prod_{k=0}^{T-1} R(k) P(k) \\
& +\frac{\lambda}{\nu} \sum_{k=0}^{T-1} \widetilde{h}(k)^{\prime} \prod_{l=k+1}^{T-1} R(l) P(l) .
\end{aligned}
$$

Noting that $E(V(T))=\sum_{j=1}^{m} E\left[V(T) 1_{\{\theta(T)=j\}}\right]=$ $\widetilde{q}(T)^{\prime} e$, we have from (9) that

$$
E(V(T))=\widetilde{q}(0)^{\prime} \mathcal{Z}(0)+\frac{\lambda}{\nu} \sum_{k=0}^{T-1} \widetilde{h}(k)^{\prime} \mathcal{Z}(k+1) .
$$

Substituting $a$ and $b$ from (16) into (22), we get (18). Now we proceed to derive the expression for the variance of the final wealth as in (19). Taking square on both sides of (20) yields

$$
\begin{aligned}
V(t+1)^{2}= & \left(A_{\theta(t)}(t)^{2}-2 A_{\theta(t)}(t) B_{\theta(t)}(t)^{\prime} \phi_{\theta(t)}(t)^{-1}\right. \\
& \cdot \varphi_{\theta(t)}(t)+\varphi_{\theta(t)}(t)^{\prime} \phi_{\theta(t)}(t)^{-1} \\
& \left.\cdot B_{\theta(t)}(t) B_{\theta(t)}(t)^{\prime} \phi_{\theta(t)}(t)^{-1} \varphi_{\theta(t)}(t)\right) V(t)^{2} \\
& -\left[\left(A_{\theta(t)}(t)-B_{\theta(t)}(t)^{\prime} \phi_{\theta(t)}(t)^{-1} \varphi_{\theta(t)}(t)\right)\right. \\
& \left.\cdot \frac{\mathcal{E}_{\theta(t)}[Z(t+1)]}{\mathcal{E}_{\theta(t)}[K(t+1)]} B_{\theta(t)}(t)^{\prime} \phi_{\theta(t)}(t)^{-1} \chi_{\theta(t)}(t)\right] \\
& \cdot V(t)+\frac{1}{4}\left(\frac{\mathcal{E}_{\theta(t)}[Z(t+1)]}{\mathcal{E}_{\theta(t)}[K(t+1)]}\right)^{2} \chi_{\theta(t)}(t)^{\prime} \\
& \cdot \phi_{\theta(t)}(t)^{-1} B_{\theta(t)}(t) B_{\theta(t)}(t)^{\prime} \phi_{\theta(t)}(t)^{-1} \\
& \cdot \chi_{\theta(t)}(t) .
\end{aligned}
$$

Let $g_{j}(t)=E\left(V(t)^{2} 1_{\{\theta(t)=j\}}\right)$. From (Costa et al., 2005) and (23) it follows that

$$
\begin{aligned}
g_{j}(t+1) & =\sum_{i=1}^{m} p_{i j} Q_{i}(t) g_{i}(t) \\
& +\frac{1}{4} \sum_{i=1}^{m} p_{i j} \pi_{i}(t)\left(\frac{\mathcal{E}_{i}[Z(t+1)]}{\mathcal{E}_{i}[K(t+1)]}\right)^{2} \beta_{i}(t) .
\end{aligned}
$$

Defining $\widetilde{g}(t)=\left(g_{1}(t), \ldots, g_{m}(t)\right)^{\prime}$ and from (15) we can rewrite (24) as

$$
\widetilde{g}(t+1)^{\prime}=\widetilde{g}(t)^{\prime} Q(t) P(t)+\left(\frac{\lambda}{\nu}\right)^{2} \widetilde{r}(t)^{\prime}
$$

and thus

$$
\begin{aligned}
\widetilde{g}(T)^{\prime} & =\widetilde{g}(0)^{\prime} \prod_{k=0}^{T-1} Q(k) P(k) \\
& +\left(\frac{\lambda}{\nu}\right)^{2} \sum_{k=0}^{T-1} \widetilde{r}(k)^{\prime} \prod_{l=k+1}^{T-1} Q(l) P(l) .
\end{aligned}
$$

Recalling that $E\left(V(T)^{2}\right)=\widetilde{g}(T)^{\prime} e$, we have from (9) that

$$
E\left(V(T)^{2}\right)=\widetilde{g}(0)^{\prime} \mathcal{K}(0)+\left(\frac{\lambda}{\nu}\right)^{2} \sum_{k=0}^{T-1} \widetilde{r}(k)^{\prime} \mathcal{K}(k+1)
$$

Replacing $c$ and $d$ from (17) into (25) and recalling that $d=$ $\frac{b}{2}$, we obtain the expected square value of the final wealth as $E\left(V(T)^{2}\right)=c+\left(\frac{\lambda}{\nu}\right)^{2} \frac{b}{2}$.

Hence, from (18), we get that

$$
\begin{aligned}
\operatorname{Var}(V(T)) & =E\left(V(T)^{2}\right)-E(V(T))^{2} \\
& =c-a^{2}-\left(\frac{\lambda}{\nu}\right) \frac{b}{2}\left(4 a-\left(\frac{\lambda}{\nu}\right)(1-2 b)\right),
\end{aligned}
$$

which is the desired equation (19) for the variance of the final wealth.

\section{SOLUTION OF THE PROBLEMS}

We solve in this section the three mean-variance problems posed in Section 2 and explicitly derive an expression for the efficient frontier. Let $\Pi\left(P 1\left(\sigma^{2}\right)\right), \Pi(P 2(\epsilon)), \Pi(P 3(\nu))$ and $\Pi(A(\lambda, \nu))$ denote, respectively, the set of optimal solutions for problems $P 1\left(\sigma^{2}\right), P 2(\epsilon), P 3(\nu)$ and $A(\lambda, \nu)$. We recall the following results, proved in ( $\mathrm{Li}$ and $\mathrm{Ng}, 2000)$.

Proposition 1 If $u \in \Pi(P 3(\nu))$ then $u \in \Pi(A(\lambda, \nu))$ with $\lambda=1+2 \nu E\left(V^{u}(T)\right)$. 
Proposition 2 Suppose that $\nu \geq 0$ and $u \in \Pi(P 3(\nu))$. a) If $\operatorname{Var}\left(V^{u}(T)\right)=\sigma^{2}$ then $u \in \Pi\left(P 1\left(\sigma^{2}\right)\right)$. b) If $E\left(V^{u}(T)\right)=\epsilon$ then $u \in \Pi(P 2(\epsilon))$.

Next we present the solution of problems $P 3(\nu), P 1\left(\sigma^{2}\right)$ and $P 2(\epsilon)$.

Theorem 3 An optimal strategy $u$ for problems $P 3(\nu)$, $P 1\left(\sigma^{2}\right)$ and $P 2(\epsilon)$ is given by $(10)$ with $K_{i}(t)$ and $Z_{i}(t)$ as in (8) and $\lambda=\frac{1+2 \nu a}{1-2 b}$. For problems $P 1\left(\sigma^{2}\right)$ and $P 2(\epsilon)$, $\nu$ is given by

$$
\nu=\left\{\begin{array}{ll}
\sqrt{\frac{b}{2 a^{2}-2(1-2 b)\left(c-\sigma^{2}\right)}} & \text { for problem } P 1\left(\sigma^{2}\right) \\
\frac{b}{\epsilon(1-2 b)-a} & \text { for problem } P 2(\epsilon)
\end{array} .\right.
$$

The expected value and variance of the terminal wealth are, respectively, given by

$$
\begin{aligned}
& E(V(T))=\frac{a \nu+b}{\nu(1-2 b)} \\
& \operatorname{Var}(V(T))=c-\frac{a^{2}}{(1-2 b)}+\frac{b}{2 \nu^{2}(1-2 b)} .
\end{aligned}
$$

Finally the efficient frontier of the multi-period meanvariance problem with regime switching is given by:

$$
\begin{aligned}
\operatorname{Var}(V(T)) & =\left(c-\frac{a^{2}}{(1-2 b)}\right) \\
& +\frac{(1-2 b)}{2 b}\left(E(V(T))-\frac{a}{(1-2 b)}\right)^{2}
\end{aligned}
$$

Proof: From Proposition 1 if $u \in \Pi(A(\lambda, \nu))$ is such that $\lambda=1+2 \nu E\left(V^{u}(T)\right)$ then $u \in \Pi(P 3(\nu))$. Combining (18) and Proposition 1, we have

$$
\begin{aligned}
\lambda & =1+2 \nu E\left(V^{u}(T)\right) \\
& =1+2 \nu\left(a+\frac{\lambda}{\nu} b\right) \Longrightarrow \lambda=\frac{1+2 \nu a}{1-2 b} .
\end{aligned}
$$

Substituting (29) into (18) leads to (26). Replacing (29) into (19), we have (27). For problem $P 1\left(\sigma^{2}\right)$ we have from Proposition 2 and (27) that

$$
\sigma^{2}=\operatorname{Var}\left(V^{u}(T)\right)=c-\frac{a^{2}}{(1-2 b)}+\frac{b}{2 \nu^{2}(1-2 b)},
$$

and thus $\nu=\sqrt{\frac{b}{2 a^{2}-2(1-2 b)\left(c-\sigma^{2}\right)}}$. For problem $P 2(\epsilon)$ we have from Proposition 2 and (26) that

$$
\epsilon=E\left(V^{u}(T)\right)=\frac{a \nu+b}{\nu(1-2 b)},
$$

and thus $\nu=\frac{b}{\epsilon(1-2 b)-a}$. Finally by combining (26) and (27) and eliminating the parameter $\nu$, we have (28), completing the proof.
Notice that the efficient frontier equation has an hyperbolic shape with center $\left(0, \frac{a}{(1-2 b)}\right)$. The minimum variance of the terminal wealth is given by $\operatorname{Var}(V(T))_{\min }=c-\frac{a^{2}}{(1-2 b)}$ and the expected terminal wealth associated to this portfolio is $E(V(T))_{\min }=\frac{a}{(1-2 b)}$.

Remark 1 Comparing Theorems 1 and 2, in which the financial market consists only of risky assets, with equations (20) and (34) to (38) in (Çakmak and Özekici, 2006), we can see that the hypothesis of the existence of a riskless asset depending on the Markov chain, as in (Çakmak and Özekici, 2006), produces no simplification on the final expressions for the control law and for the expected value and variance of the final wealth.

\section{THE SPECIAL CASE WITH ONE RISK- LESS ASSET}

Let us investigate now the special case in which one of the assets is riskless, that is, it has no volatility and it is unaffected by the Markov chain. We assume the asset $i=0$ as the riskless one. The price of the risk-free asset evolves as in (1), with $\sigma_{0}(t, i)=0$ and $\mu_{0}(t, i)=r_{f}(t)$ for all $t$ and $i \in M$. The existence of a riskless asset allows us to simplify some equations in our model. Indeed, for each $i \in \mathcal{M}$ and $t=0, \ldots, T$, it follows that

$$
\begin{aligned}
\bar{A}_{i}(t) & =\bar{A}(t)=1+r_{f}(t), \\
\delta_{i}(t) & =E\left(A_{i}(t)^{2}\right)=\bar{A}(t)^{2}, \\
\varphi_{i}(t)^{\prime} & =E\left(A_{i}(t) B_{i}(t)^{\prime}\right) \\
& =\bar{A}(t) E\left(B_{i}(t)^{\prime}\right) \\
& =\bar{A}(t) \chi_{i}(t)^{\prime}, \\
R_{i}(t) & =\bar{A}(t)\left(1-\beta_{i}(t)\right)
\end{aligned}
$$

and

$$
Q_{i}(t)=\bar{A}(t) R_{i}(t)=\bar{A}(t)^{2}\left(1-\beta_{i}(t)\right)
$$

Set

$$
\begin{gathered}
\rho(t)=\prod_{k=t}^{T-1} \bar{A}(k), \\
\widetilde{\beta}(t)=\operatorname{diag}\left(\beta_{i}(t)\right),
\end{gathered}
$$

and

$$
\widetilde{\mathcal{Z}}(t)=\left[\prod_{k=t}^{T-1}(I-\widetilde{\beta}(k)) P(k)\right] e .
$$

We have that

$$
\begin{gathered}
\mathcal{K}(t)=\rho(t) \mathcal{Z}(t) \\
\widetilde{h}(t)^{\prime}=\frac{1}{2 \rho(t+1)} \pi(t)^{\prime} \widetilde{\beta}(t) P(t),
\end{gathered}
$$




$$
\mathcal{Z}(t)=\rho(t) \widetilde{\mathcal{Z}}(t)
$$

and

$$
\begin{gathered}
a=\rho(0) V(0) \pi(0)^{\prime} \widetilde{\mathcal{Z}}(0), \\
c=(\rho(0) V(0))^{2} \pi(0)^{\prime} \widetilde{\mathcal{Z}}(0),
\end{gathered}
$$

so that $\frac{a^{2}}{c}=\pi(0)^{\prime} \widetilde{\mathcal{Z}}(0)$. We have the following result.

Theorem 4 An optimal strategy u for problems P3 $(\nu)$, $P 1\left(\sigma^{2}\right)$ and $P 2(\epsilon)$ is given by

$$
u(t)=-\phi_{\theta(t)}(t)^{-1} \chi_{\theta(t)}(t)\left(\bar{A}(t) V(t)-\frac{\lambda}{2 \nu \rho(t+1)}\right)
$$

with $\lambda$ and $\nu$ as in Theorem 3. Moreover, for this optimal control law, we have that $E(V(T))$ satisfies (26) and $\operatorname{Var}(V(T))=\frac{b}{2 \nu^{2}(1-2 b)}$. The efficient frontier equation is given by

$$
\operatorname{Var}(V(T))=\frac{(1-2 b)}{2 b}\left(E(V(T))-\frac{a}{(1-2 b)}\right)^{2} .
$$

Proof: Suppose that $c=a^{2} /(1-2 b)$. Then the result is a straightforward consequence of Theorem 3 observing that in (10),

$$
\frac{\mathcal{E}_{i}[Z(t+1)]}{\mathcal{E}_{i}[K(t+1)]}=-\frac{\lambda}{\nu \rho(t+1)} .
$$

Remains to show that $c=a^{2} /(1-2 b)$. In order to show that we show by induction on $\ell=0, \ldots, T$ that

$$
\pi(0)^{\prime} \widetilde{\mathcal{Z}}(0)+\sum_{t=0}^{\ell-1} \pi(t)^{\prime} \widetilde{\beta}(t) P(t) \widetilde{\mathcal{Z}}(t+1)=\pi(\ell)^{\prime} \widetilde{\mathcal{Z}}(\ell) .
$$

Clearly (32) holds for $\ell=0$. Suppose it holds for $\ell$. Then recalling that $\widetilde{\mathcal{Z}}(\ell)=[(I-\widetilde{\beta}(\ell)) P(\ell)] \widetilde{\mathcal{Z}}(\ell+1)$, we have from (32) that

$$
\begin{aligned}
& \pi(0)^{\prime} \widetilde{\mathcal{Z}}(0)+\sum_{t=0}^{\ell} \pi(t)^{\prime} \widetilde{\beta}(t) P(t) \widetilde{\mathcal{Z}}(t+1) \\
& =\pi(\ell)^{\prime} \widetilde{\mathcal{Z}}(\ell)+\pi(\ell)^{\prime} \widetilde{\beta}(\ell) P(\ell) \widetilde{\mathcal{Z}}(\ell+1) \\
& =\pi(\ell)^{\prime}(P(\ell)-\widetilde{\beta}(\ell) P(\ell)+\widetilde{\beta}(\ell) P(\ell)) \widetilde{\mathcal{Z}}(\ell+1) \\
& =\pi(\ell+1)^{\prime} \widetilde{\mathcal{Z}}(\ell+1)
\end{aligned}
$$

since $\pi(\ell)^{\prime} P(\ell)=\pi(\ell+1)^{\prime}$, showing (32). Noting that $\widetilde{\mathcal{Z}}(T)=e$ so that $\pi(T)^{\prime} \widetilde{\mathcal{Z}}(T)=1$, it follows from (32) that

$$
\begin{aligned}
2 b & =\sum_{t=0}^{T-1} \pi(t)^{\prime} \widetilde{\beta}(t) P(t) \widetilde{\mathcal{Z}}(t+1) \\
& =1-\pi(0)^{\prime} \widetilde{\mathcal{Z}}(0) \\
& =1-\frac{a^{2}}{c}
\end{aligned}
$$

completing the proof.
As a consequence of the existence of a risk-free asset, the minimum variance of the final wealth is zero with corresponding expected value of the terminal wealth given by $E(V(T))_{\min }=\frac{a}{(1-2 b)}$.

Remark 2 It should be noticed that the results of Theorems 3 and 4 coincide with those in ( $\mathrm{Li}$ and $\mathrm{Ng}, 2000)$ for the case in which there are no switching parameters. Moreover, it is worth pointing out the difference between the expressions for the control strategy and for the expected value and variance of the final wealth we find here considering the case in which there exists a riskless asset free of any source of uncertainty, and those expressions derived in (Çakmak and Özekici, 2006), in which the risk free security depends on the Markov chain.

\section{NUMERICAL EXAMPLES}

In this section we compare the model proposed in this paper with the one presented in ( $\mathrm{Li}$ and $\mathrm{Ng}, 2000)$. The first example is the case in which all assets are risky while the second one includes a risk-free asset.

Example 1 Consider the case in which an investor with one unit wealth and an investment horizon $T=4$ has to allocate his possession among four risky assets in order to maximize his expected final wealth $E(V(4))$, while keeping the variance of the terminal wealth not exceeding 2 , i.e., $\sigma^{2}(4) \leq 2$. To simplify, we assume the multi-period process as stationary. To determine the market trends we choose the S\&P 500 Index (SPX) and, for the risky assets, we picked the four stocks which have more weight in this index: General Eletric (GE), Exxon Mobil (XOM), Citigroup (C) and Microsoft (MSFT). The daily closing prices of these stocks, starting from 2000 until the end of 2004, are used to estimate the mean and variance of them. In this example, there are two major trends: up- or down-trend, i.e., $\mathcal{M}=\{1,2\}$, respectively. To identify which mode is leading the market we used a moving average. Whenever the monthly closing price of the index is above its three period moving average, we define that month as an up-trend and whereas the monthly closing price of the index is bellow its three period moving average, we define the period as a down-trend. To estimate the mean return $\mu(t, \theta(t))$ and covariance matrix $\sigma^{2}(t, \theta(t))$ of the assets we follow an approach similar to that adopted in (Zhang, 2000). From the daily closes of the stocks from 2000 until 2004, we obtain the vector of yearly mean returns (252 trading days) and yearly covariance matrix for each market mode and for $t=0,1,2,3$, respectively, as $\mu(t, 1)=(25.4 \%, 24.4 \%, 70.8 \%, 19.8 \%)^{\prime}$, 


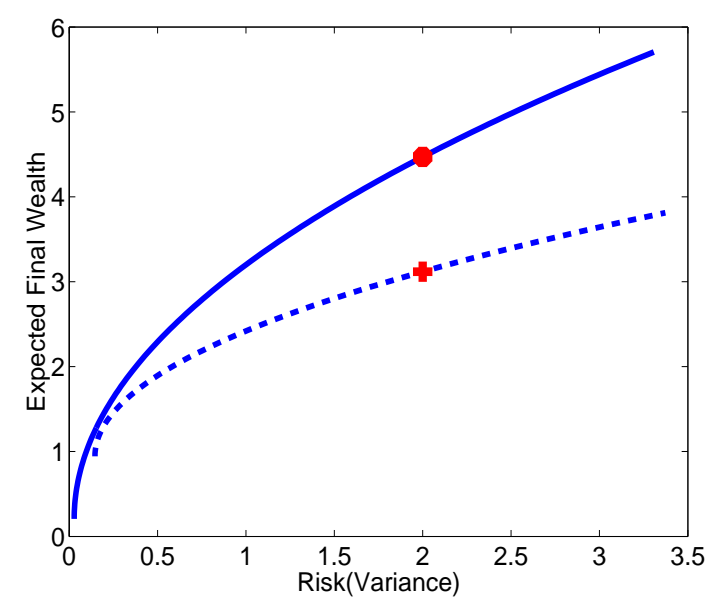

Figure 1: Efficient frontier

$$
\begin{gathered}
\mu(t, 2)=(-26.1 \%,-5.4 \%,-31.0 \%,-38.7 \%)^{\prime}, \text { and } \\
\sigma^{2}(t, 1)=\left[\begin{array}{cccc}
8.9 & 1.7 & 5.6 & 3.9 \\
1.7 & 5.2 & 2.2 & 1.3 \\
5.6 & 2.2 & 9.0 & 4.0 \\
3.9 & 1.3 & 4.0 & 12.7
\end{array}\right] / 100, \\
\sigma^{2}(t, 2)=\left[\begin{array}{cccc}
14.1 & 3.9 & 9.5 & 8.0 \\
3.9 & 7.4 & 3.9 & 3.7 \\
9.5 & 3.9 & 15.7 & 8.2 \\
8.0 & 3.7 & 8.2 & 19.6
\end{array}\right] / 100 .
\end{gathered}
$$

As the number of up-trend days was almost $50 \%$ of the total days, we choose $p_{i j}=0.5, i, j \in \mathcal{M}$, as the transition probabilities. From (28), the mean-variance efficient frontier equation is given as follows: $\operatorname{Var}(V(4))=0.029+0.108$. $(E(V(4))-0.207)^{2}$. For the maximum selected risk level $\left(\sigma^{2}(4)=2\right)$, the corresponding expected final wealth in the efficient frontier is $E(V(4))=4.47$. We implemented the model proposed in ( $\mathrm{Li}$ and $\mathrm{Ng}, 2000)$ to compare with the present one. To estimate the mean return and covariance matrix of the assets we use the whole period of the above sample (2000 - 2004) without separating the trend periods, obtaining for $t=0,1,2,3, \mu(t)=(-4.2 \%, 8.1 \%, 7.6 \%,-14.2 \%)^{\prime}$ and

$$
\sigma^{2}(t)=\left[\begin{array}{cccc}
11.6 \% & 2.9 \% & 7.6 \% & 6.1 \% \\
2.9 \% & 6.3 \% & 3.1 \% & 2.5 \% \\
7.6 \% & 3.1 \% & 12.5 \% & 6.2 \% \\
6.1 \% & 2.5 \% & 6.2 \% & 16.4 \%
\end{array}\right]
$$

For this case, the mean-variance efficient frontier equation is given by $\operatorname{Var}(V(4))=0.15+0.39 \cdot(E(V(4))-0.94)^{2}$. The expected terminal wealth corresponding to the selected risk level $\left(\sigma^{2}(4)=2\right)$ is $E(V(4))=3.12$. In Fig. 1 we can see the efficient frontier for both cases. The continuous line represent the efficient frontier for the case with regime switching while the dotted line is the efficient frontier following the model proposed in ( $\mathrm{Li}$ and $\mathrm{Ng}, 2000)$. The expected terminal wealth corresponding to the selected risk level is pointed out in Fig. 1 by an asterisk and by a cross, respectively for the case with and without Markov switching parameters. Therefore, in this example, for the same accepted final risk level $\left(\sigma^{2}(4)=2\right)$, the first case delivered an expected final wealth above the case without jumps. This outcome illustrates the advantage of the present model to better capture the market movements.

Example 2 Now we examine the situation with the existence of a riskless asset. In this example we use the same assets presented above plus a risk-free asset. The riskless asset is represented by the two years Fed Fund rate (GT2). Although this interest rate also changes following the market movements, we considered it constant and equal to its average in the period: $r_{f}(t)=3.3 \%$, for $t=0,1,2,3$. The objective is the same as above and we use the same assets and conditions as presented in the former example, just adding the riskless asset. We get the following meanvariance efficient frontier equation: $\operatorname{Var}(V(4))=0.009$. $(E(V(4))-1.14)^{2}$. Now, considering the model presented in ( $\mathrm{Li}$ and $\mathrm{Ng}, 2000$ ), with the same data as in the last example and adding the riskless asset $i=0$ we get the following efficient frontier equation: $\operatorname{Var}(V(4))=0.30$. $(E(V(4))-1.14)^{2}$. The expected terminal wealth in each situation is $E(V(4))=16.41$ and $E(V(4))=3.72$, respectively. These two points are plotted in Fig. 2 by an asterisk and a cross, respectively for the case with and without regime switching. In Fig. 2, we plotted the two efficient frontier equations. As in the former example, the outcome of the case with the key parameters modulated by a Markov chain (solid line) beat by far the case without this feature (dotted line).

\section{CONCLUSIONS}

In this paper we extended the work of (Çakmak and Özekici, 2006) by studying a discrete-time multi-period meanvariance portfolio selection problem subject to Markovian jumps in the parameters. An optimal investment strategy for this mean-variance problem was analytically derived in a closed form. We showed that this optimal policy depends upon a set of interconnected Riccati difference equations presented in (8). As a result, an explicit expression for the efficient frontier was identified. Our results coincide with those in ( $\mathrm{Li}$ and $\mathrm{Ng}, 2000$ ) for the case in which there are no switching parameters. The advantage of this model is to better respond to drastic movements of the market as a result of stress situations or discontinuity changes due to external factors.

Finally it is worth mentioning that extensions of the for- 


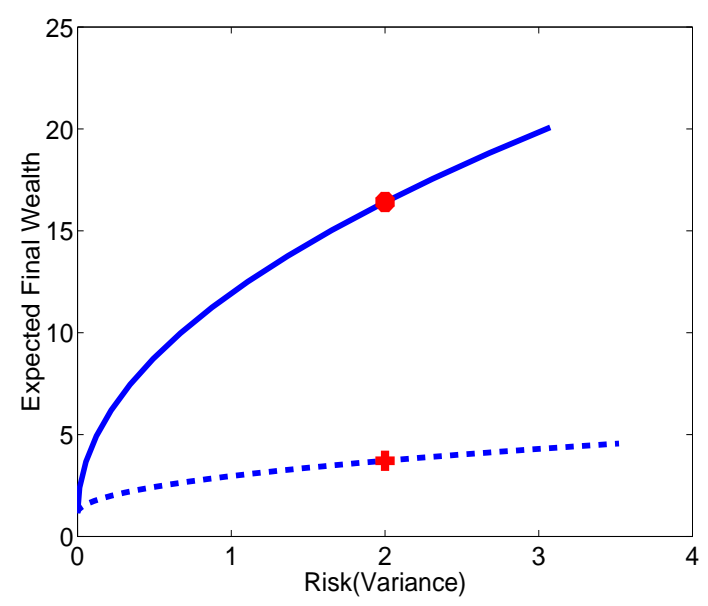

Figure 2: Efficient frontier with a riskless asset

mulation presented here could be obtained by considering a generalized multi-period mean variance portfolio optimization problem with Markov switching parameters, as studied in (Costa and Araujo, 2008). The generalized multi-period mean-variance problem can be seen as an stochastic control problem in which the objective function is formed by a weighted sum of a linear combination of the expected value and square of the expected value of the wealth, and the expected value of the square of the wealth. A great variety of mean-variance models with intermediate restrictions and/or intermediate costs in the objective function can be derived from this generalized formulation. The usefulness of adopting this kind of criterion is that in several situations investor managers have to report their portfolio's return in a periodic basis to their beneficiaries, clients or to governmental authorities, so that intermediate performances are as important as the final one. Moreover intermediate restrictions could also be included in this formulation (see also (Costa and Nabholz, 2007)). Therefore more traditional mean-variance problems, which regards the performance only at the final value, would not be the most appropriate for these situations. On the other hand the price one pays by adopting this more general approach is that a solution for the problem usually requires a numerical procedure based on a Lagrangian dual minimization problem (see (Zhu et al., 2004), (Costa and Araujo, 2008)).

\section{ACKNOWLEDGMENT}

The first author received financial support from $\mathrm{CNPq}$ (Brazilian National Research Council), grant 304866/03-2 and FAPESP (Research Council of the State of São Paulo), grant 03/06736-7. The authors would like to express their gratitude to the associate editor and referees for their sugges- tions and helpful comments.

\section{REFERENCES}

Bauerle, N. and Rieder, U. (2004). Portfolio optimization with Markov-modulated stock prices and interest rates, IEEE Trans. Autom. Control 49: 442-447.

Çakmak, U. and Özekici, S. (2006). Portfolio optimization in stochastic markets, Math. Methods Oper. Research 63: $151-168$.

Costa, O. L. V. and Araujo, M. V. (2008). A generalized multi-period mean-variance portfolio optimization with Markov switching parameters, Automatica, to appear .

Costa, O. L. V., Fragoso, M. D. and Marques, R. P. (2005). Discrete-Time Markov Jump Linear Systems, SpringerVerlag.

Costa, O. L. V. and Nabholz, R. (2007). Multiperiod mean-variance optimization with intertemporal restrictions, Journal of Optimization Theory and Applications 134: 257-274.

Li, D. and Ng, W. (2000). Optimal dynamic portfolio selection: Multi-period mean-variance formulation, Math. Finance 10: 387-406.

Markowitz, H. (1952). Portfolio selection, J. Finance 7: 7791.

Yin, G. and Zhou, X. Y. (2004). Markowitz's meanvariance portfolio selection with regime switching: From discrete-time models to their continuous-time limits, IEEE Trans. Autom. Control 49: 349-360.

Zhang, Q. (2000). Stock trading: An optimal selling rule, SIAM J. Control Optim. 40: 64-87.

Zhou, X. Y. and Yin, G. (2003). Markowitz's meanvariance portfolio selection with regime switching: A continuous-time model, SIAM J. Control Optim. 42: $1466-1482$.

Zhu, S.-S., Li, D. and Wang, S.-Y. (2004). Risk control over bankruptcy in dynamic portfolio selection: A generalized mean-variance formulation, IEEE Trans. Autom. Control 49: 447-451. 